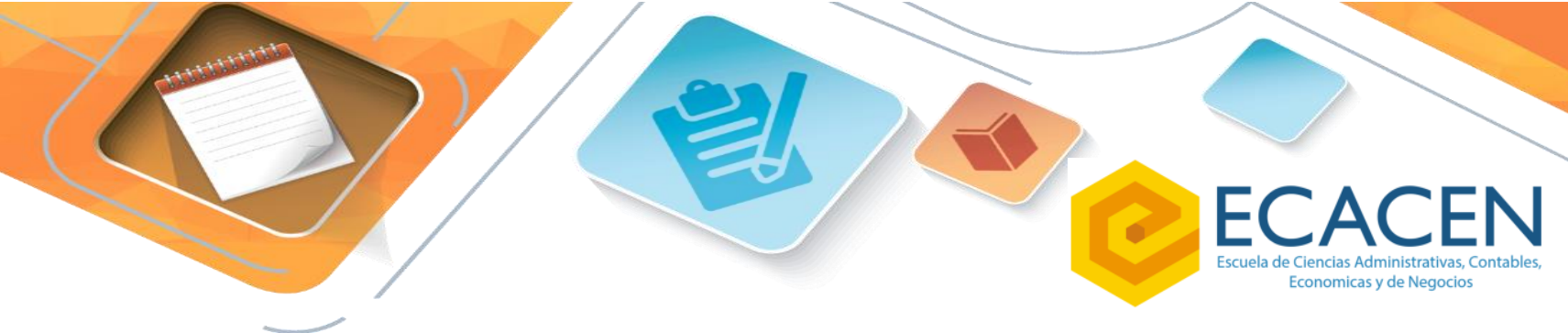

\title{
CONSIDERACIONES GENERALES SOBRE LA FORMULACIÓN Y EVALUACIÓN DE PROYECTOS DE INVERSIÓN
}

\author{
NOMBRE DE AUTORES \\ Mg. Martha Lucía Fuertes Díaz ${ }^{1}$ \\ Yudith Caicedo ${ }^{2}$ \\ Alicia Cristina Silva ${ }^{3}$
}

\section{RESUMEN}

Para apoyar al inversionista en el proceso de toma de decisiones de inversión se requieren estudios especializados, entre los cuales sobresale la Formulación y Evaluación de proyectos de Inversión, por ello el objetivo de este documento pretende orientar sobre la importancia de este tipo de estudios y establecer las fuentes de información que acoge, las etapas en que se desarrolla y que facilitan al inversionista su proceso de toma de decisiones en consideración de la rentabilidad y riesgo implícito en su decisión. Este documento aporta claridad sobre lo que se considera proyecto de inversión, la clasificación de los proyectos, las etapas que agotan estos estudios conocidos como ciclo de vida de los proyectos, la clasificación de los proyectos, para finalmente describir de manera general cada uno de estos estudios y que constituyen la formulación y evaluación de proyectos de inversión. En la medida en que se van desarrollando cada uno de los estudios, cuyas bases se sustentan en información primaria y secundaria, se va acumulando una base muy importante de conocimiento sobre el proyecto que se propone desde los enfoques de mercado, técnico, financiero, económico y social que conducen a la determinación de la factibilidad del proyecto y se constituyen en una base fundamental para la toma de decisiones de inversión.

\footnotetext{
${ }^{1}$ Docente de la Universidad Nacional Abierta y a Distancia, Contadora Pública, Magister en Administración con Énfasis en Finanzas Corporativas, Especialista en Finanzas con concentración en Finanzas Avanzadas, $\mathrm{PhD}($ C) Administración Gerencial. Correo electrónico de contacto martha.fuertes@unad.edu.co

${ }^{2}$ Administradora de Empresas, especialista en Gestión del Talento Humano en las Organizaciones, magister en Administración de Organizaciones, yudith.caicedo@unad.edu.co.

${ }^{3}$ Administradora de empresas, Especialista en Pedagogía para el Desarrollo del Aprendizaje Autónomo, Magister en Sistemas de Calidad y Productividad, PhD en Administración. Docente Ocasional UNAD - CCAV Pasto, grupo de Investigación Sindamanoy, correo electrónico alicia.silva@unad.edu.co, C.C. 59823146 de Pasto, Código ORCID orcid.org/0000-0002-9329-0777
} 


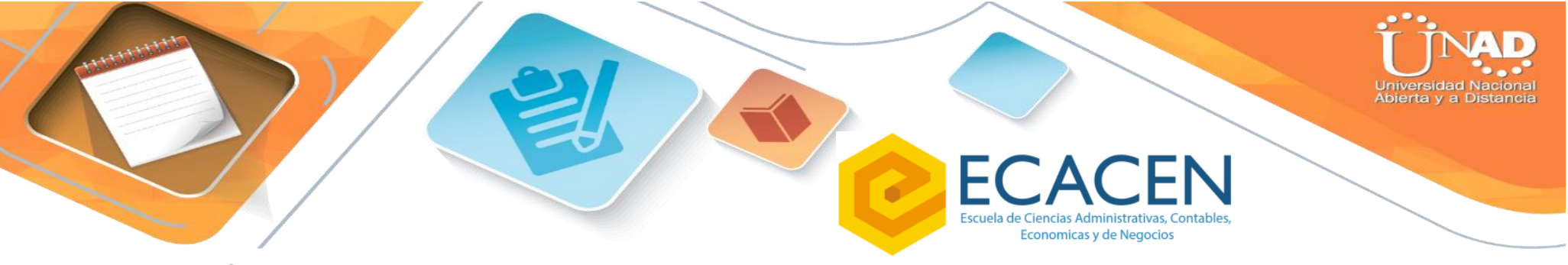

Palábras Clave: Formulación de proyectos; Proyectos de inversión; Evaluación de proyectos.

\begin{abstract}
To support the investor in the investment decision-making process, specialized studies are required, among which the Formulation and Evaluation of Investment projects stands out, for this reason the objective of this document is to guide on the importance of this type of studies and establish the information sources that it receives, the stages in which it is developed and that facilitate the investor's decision-making process in consideration of the profitability and risk implicit in its decision. To the extent that each of the studies is developed, whose information bases link primary and secondary information, a very important base of knowledge is accumulated on the proposed project from the market, technical, financial, and economic approaches. and social that lead to the determination of the feasibility of the project and constitute a fundamental basis for making investment decisions. This document provides clarity on what is considered an investment project, the classification of the projects, the stages that exhaust these studies known as the life cycle of the projects, the classification of the projects, to finally describe in a general way each one of these studies and that constitute the formulation and evaluation of investment projects.
\end{abstract}

Keywords: Projects formulation; Investment projects; Project evaluation. 


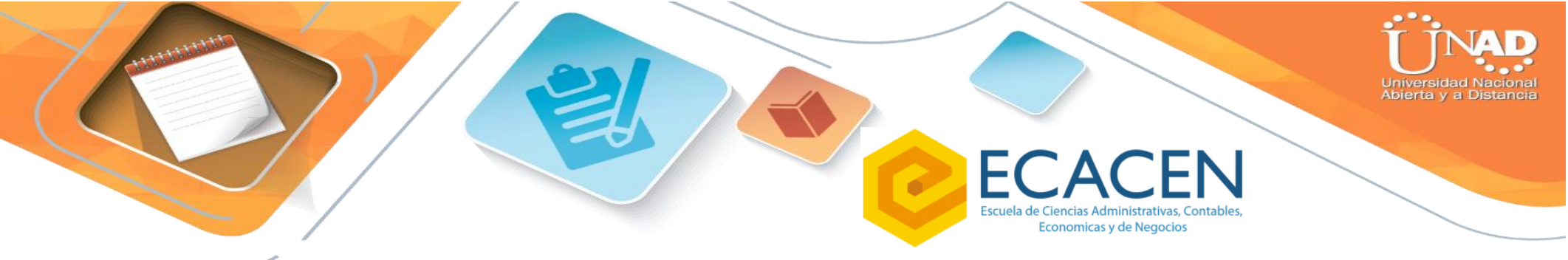

\section{INTRODUCCIÓN}

En los estudios de factibilidad que soportan las propuestas de proyectos de inversión es indispensable realizar análisis y estudios que conducen al agrupamiento de un cúmulo de información sobre el proyecto que se propone, que además de soportar el proceso de toma de decisión sobre la conveniencia de la inversión para el inversionista, también aporta en la gestión de los riesgos que a futuro enfrentarán estos proyectos, cuando la decisión sea realizarlos.

Para la determinación de la factibilidad de un proyecto de inversión, los estudios que lo soportan aportan al proceso de toma de decisiones sobre la conveniencia o no para su realización; pero también aportan en los análisis de los niveles de riesgos implícitos en este tipo de inversiones, por el conocimiento ex-ante que se logra y con el que se desarrolla mayor nivel de preparación para enfrentar el contexto y situaciones futuras que enfrentaran los nuevos emprendimientos.

Además, se debe considerar que son múltiples los enfoques en que se desarrollan los proyectos, por ello es importante tener claridad sobre su clasificación para el desarrollo futuro de los estudios de factibilidad, pues el enfoque de estos estudios va a depender si se trata de un proyecto Económico o Social según su carácter, o de un proyecto de producción de bienes, prestación de servicios o Infraestructura según su categoría; o si se trata de un proyecto agropecuarios, industriales, comercial de servicio, de infraestructura social y económica, etc., base sobre la cual se desarrollan los estudios de prefactibilidad y factibilidad que permiten determinar su viabilidad.

Los anteriores estudios, cada uno a la vez, deben aportar en la conclusión sobre la viabilidad desde el punto de vista de mercado, técnico y organizacional para el proyecto, que deben ser complementados con el estudio financiero y la evaluación económica del proyecto, estudios que permitirán concluir sobre la factibilidad del proyecto y establecer con mayor nivel de seguridad y menor nivel de riesgo, la conveniencia de llevar a la realidad el proyecto de inversión analizado.

Generalmente, el estudio financiero del proyecto, es el último estudio en realizarse debido a que requiere de la información lograda en el estudio de mercado, técnico y organizacional, y a partir de esta información establecida en términos cuantitativos determinar la factibilidad del proyecto a partir de su viabilidad económica y social. 


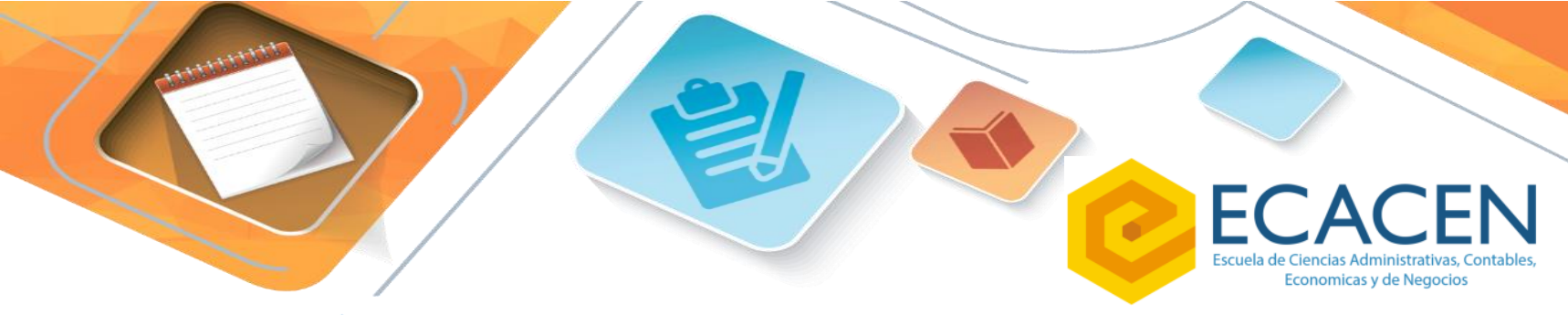

\section{DESARROLLO}

Los inversionistas que analizan entre diversas alternativas u oportunidades de inversión consideran dos aspectos fundamentalmente al momento de tomar su decisión, que se centran en el análisis de la rentabilidad y del riesgo, que implica cada alternativa. En este propósito aportan los estudios de formulación y evaluación de proyectos de inversión que ofrecen información que cubre cada propuesta, en todos los aspectos que la integran y desde varios enfoques que permite concluir sobre la factibilidad del proyecto analizado y cuya información relevante permite que los inversionistas puedan seleccionar entre las alternativas de inversión, aquel o aquellos proyectos que cumplan con sus expectativas de rentabilidad y el nivel de riesgo, a partir de lo cual estarían dispuestos a invertir (Arboleda, 2012).

Pero antes de profundizar en los estudios que se deben desarrollar en un proceso de formulación y evaluación de proyectos de inversión, se debe partir de la conceptualización de lo que es un proyecto, que corresponde a:

Proyecto es una propuesta ordenada de acciones que pretende la solución o reducción de la magnitud de un problema que afecta a un individuo o grupo de individuos y en la cual se plantea la magnitud, característica, tipos y períodos de los recursos requeridos para completar la solución propuesta, dentro de las limitaciones técnicas, sociales, económicas y políticas en las cuales el proyecto se desenvolverá. (Contreras, 1997, p.24)

Avanzando en la conceptualización, se sigue con la definición de proyecto de inversión, para lo cual se seleccionan un par de definiciones, cada una de ellas con un enfoque planteado de manera particular con iguales implicaciones generales.

El proyecto de Inversión comprende todo el análisis, juzgamiento y evaluación de factores, para determinar las ventajas que se derivan de utilizar recursos económicos en la producción de un determinado bien o servicio, a través de una organización empresarial capaz de acometer este propósito. (Unisur citado por Contreras, 1997, p.25)

Igualmente, para la definición de proyecto de inversión se encuentran múltiples propuestas, entre las cuales se resalta la realizada por el Instituto Latinoamericano de Planificación Económica y Social (Como se citó en Pimentel, 2008):

Es el plan prospectivo de una unidad de acción capaz de materializar algún aspecto del desarrollo económico o social. Esto implica desde el punto de vista económico, proponer la producción de algún bien o la prestación de algún servicio, con el empleo de ciertas técnicas y con miras a obtener un determinado resultado o ventaja económica o social. (Pimentel, 2008, p.8) 
Sin embargo, se reconoce la definición realízada por Contreras, que se orienta más específicamente a la definición de proyectos de inversión empresarial, que generalmente también conocemos como plan de negocio o proyectos de emprendimiento:

El proyecto Empresarial comprende todo el análisis, juzgamiento y evaluación de factores, para determinar las ventajas que se derivan de utilizar recursos económicos en la producción de un determinado bien o servicio, a través de una organización empresarial capaz de acometer este propósito. (1997, p.25)

De acuerdo con las anteriores definiciones, es importante considerar que este tipo de estudios son prospectivos, lo que implica que para su desarrollo se deben considerar análisis para un amplio período de tiempo dado el impacto de largo plazo establecido para la evaluación de proyectos de inversión, que finalmente son determinantes en la creación o ampliación de empresas cuando se decide realizarlos.

Cada uno de los proyectos que se proponen se orientan en dimensiones diferentes que cubren múltiples aspectos, por lo que se establecen varias clasificaciones dependiendo de las diferentes tipologías en las cuales se enmarcan, como lo señala Contreras (1997), entre ellas se destacan las siguientes:

- Los proyectos según su carácter pueden ser económicos y sociales. Los proyectos económicos son aquellos que persiguen ánimo de lucro y deben tener la capacidad para establecer una demanda real en el mercado. Por su parte, los proyectos sociales persiguen la generación de beneficio social y no lucrativo.

- Los proyectos según su categoría pueden ser de producción de bienes, prestación de servicios y de infraestructura.

- Los proyectos de acuerdo con su actividad económica pueden ser agropecuarios, industriales, comerciales, de servicios y mineros.

En el proceso de formulación y evaluación de proyectos se agotan los períodos de gestación, implementación y operación. El período de gestación se caracteriza por corresponder a la etapa de identificación de la idea del proyecto, que debe surgir de una necesidad latente en la población objeto del estudio, que no esté satisfecha o que si bien se encuentran productos o servicios orientados a satisfacerla, el nivel de satisfacción puede ser mejorado mediante la innovación del producto o servicio (Bacca, 2013).

Se avanza en el estudio, con la recolección de información preliminar de fuentes secundarias para ello se analiza el contexto externo referente a aspectos de desarrollo económico, social y político del país, sector económico y región donde se propone el desarrollo del proyecto, además de análisis la competencia y de otras fuentes de información de entidades y organismos relacionados, que permitan aportar en la conformación del primer análisis sobre la conveniencia de llevar a cabo el proyecto; a este primer estudio también se conoce como estudio de prefactibilidad. 
Si realizado el estudio de prefactibilidad se considerando que el proyecto ofrece atractivo para su viabilidad, se pasa al siguiente estudio que se denomina estudio de factibilidad del proyecto, conformado por varios estudios, cada uno de ellos debe profundizar en la información, análisis y técnicas aplicadas para evaluar sobre su factibilidad y si cada estudio después del análisis realizado es factible da continuidad con el siguiente estudio. Los estudios de factibilidad se caracterizan por que incluyen una amplia base de información primaria que gira en torno directo al proyecto que se desarrolla; los estudios que se deben realizar son: Estudio de factibilidad de mercado, estudio de factibilidad técnico, estudio de factibilidad financiero y el estudio de la evaluación económica y social del proyecto.

Chain (2007) señala que el primer estudio que se realiza para determinar la factibilidad de un proyecto de inversión o de un emprendimiento corresponde al Estudio de Factibilidad de Mercado que se centra en análisis profundo del mercado al cual se quiere impactar con el proyecto, que analiza el producto, consumidores o demanda, competencia u oferta, precios, canales de distribución y plan de comercialización, entre otros. Estos estudios permiten avanzar con una investigación más profunda y directa del mercado objetivo para el proyecto mediante la denominada investigación de mercado para lo cual se aplican técnicas que permiten la recolección de información primaria como la encuesta y que debe conducir al establecimiento de la proyección de ventas, tanto en unidades a colocar en el mercado, el precio y que finalmente permite establecer los ingresos para el proyecto para el primer año, a partir del cual se realiza la proyección de los ingresos para los siguientes años en que se analiza la factibilidad del proyecto.

Determinada la viabilidad de mercado para el proyecto de inversión o emprendimiento, se da continuidad con el desarrollo del Estudio de Factibilidad Técnica y el Estudio Organizacional. El estudio técnico aporta significativamente en la determinación del tamaño del proyecto, su ubicación, el proceso productivo, la identificación y análisis de costos y gastos, la distribución de planta, la inversión inicial requerida y el estudio que aporta para la organización administrativa.

Los anteriores estudios, cada uno a la vez, deben aportar en la conclusión sobre la viabilidad desde el punto de vista de mercado, técnico y organizacional para el proyecto, que de ser positivo se da continuidad con el estudio financiero y la evaluación económica del proyecto que corresponde al último estudio en realizarse debido a que para su desarrollo se requiere de la información lograda en el estudio de mercado, técnico y organizacional, y a partir de esta información establecida en términos cuantitativos se logra determinar sobre la factibilidad del proyecto desde lo financiero y económico (Mille, 2004)

Este proceso pretende finalmente conducir a soportar el proceso de la toma de decisión sobre la factibilidad del proyecto y establecer con mayor nivel de seguridad y menor 
nivel de riesgo, la conveniencia de llevar a la realídad el proyecto de inversión analizado.

\section{CONCLUSIONES}

Los estudios de formulación y evaluación de proyectos de inversión aportan significativamente en dos momentos, el primero antes del desarrollo del proyecto cuando se analiza la conveniencia de llevar a cabo el proyecto que aporta al inversionista información y análisis en los que puede sustentar su proceso de toma de decisión; y para los casos en que el proyecto se desarrolla, aporta en el control durante los períodos de ejecución y operación al ofrecer información importante que permite contrastar lo planeado con la realidad que se va desarrollando.

La base sobre la cual se desarrollan los proyectos debe surgir de la identificación de una necesidad latente en la comunidad, a partir de lo cual se proponen los proyectos para satisfacer las necesidades identificadas $y$ que se constituyen en factor fundamental para el éxito.

Durante el desarrollo de los estudios de factibilidad, se analiza y profundiza en la solución del problema identificado mediante el análisis de múltiples alternativas a través de los estudios de prefactibilidad y de factibilidad, lo cual aporta información valiosa al momento de decidir sobre la de mayor conveniencia para conformar los estudios de factibilidad.

Es importante avanzar en la formulación y evaluación de proyectos a partir de la necesidad identificada en que se sustenta la propuesta de la idea del proyecto y que requiere de un primer estudio denominado de pre-factibilidad y que de su resultado permitirá avanzar en los estudios de factibilidad.

Los estudios de factibilidad deben realizarse a profundidad y aterrizando toda la información requerida al proyecto que se propone, desde varios enfoques: Mercado, Técnico, Financiero, Económico y Social.

En la medida en que se desarrolla cada estudio debe analizarse la factibilidad del proyecto y de ser positiva la respuesta se procede con el desarrollo del siguiente estudio de factibilidad, y así hasta llegar a la evaluación económica y social del proyecto, como base para determinar su factibilidad y que será para el inversionista un estudio valioso al momento de analizar sobre la conveniencia de su decisión de inversión.

Los estudios realizados en la formulación y evaluación de proyectos de inversión permiten determinar sobre la viabilidad y factibilidad del proyecto y por lo tanto su aporte es fundamental para los inversionistas para disminuir el nivel de riesgo en que incurren con su decisión. 


\section{REFERENCIAS}

- Arboleda, G. (2012). Proyectos, formulación, evaluación y control.

- Baca, G. (2013). Evaluación de proyectos. 7a.Ed. Mc Graw Hill.

- Chain, N. 2007. Proyectos de inversión formulación y evaluación. Pearson/ Prentice Hall.

- Contreras M. (1997). Formulación y evaluación de proyectos. Bogotá: Editorial Unad.

- González R. Rene (2004) Elaboración de Planes de Negocios, Mc Graw

Hill.

- Mille, J. (2004). Manual básico de elaboración y evaluación de proyectos. Recuperado

dehttp://www.colefgalicia.com/contenidos/images/stories/actualidade/manu al\%20de\%20elaboraci\%F3n\%20y\%20evaluaci\%F3n\%20de\%20proyectos\% 202004.pdf

- Pimentel, E. (2008). Formulación y evaluación de proyectos de inversión. Recuperado de https://dadospdf.com/download/libro-de-proyectosedmundo-pimentel-1-_5a4bcfc4b7d7bcab67ef99a7_pdf 